\title{
Topical Curcumin and Triamcinolone Acetonide in Recurrent Minor Aphthous Ulcers: A Pilot Trial
}

\author{
Praveena Raman ${ }^{1}$, Raghuram Pitty ${ }^{2}$, Chandrasekar Lakshmi Krithika ${ }^{3}$, SP Nehru Anand ${ }^{4}$, Gayathri Ponnusami Subramani ${ }^{5}$
}

\begin{abstract}
Aim: To evaluate the efficacy of topical curcumin and topical triamcinolone acetonide in a professional population with minor aphthous ulcers by assessing six clinical variables: site, size, pain, healing period, frequency of recurrence and number of ulcers.

Materials and methods: This randomized, parallel designed pilot trial was performed on 60 symptomatic individuals with minor aphthous ulcer. Willing participants were allocated randomly into group I and group II. Participants in group I were treated with topical curcumin and group II were treated with topical triamcinolone acetonide for a period of 6 months. All participants were blinded to the drug they received. Participants were assessed on day 1, day 3, day 5, day 7, and after healing for symptomatic reduction in pain, size, healing period, frequency of recurrence, and in the number of ulcers. Statistically, independent sample $t$ test, Chi-square test, and Log rank Kaplan-Meier survival analysis were performed. Results: Lower labial mucosa was found to be the predominant site of minor aphthous ulcer in both the groups. A gradual reduction in pain and size was noted in both the groups with statistical significance of $p$ value $<0.001$. All the ulcers in both the groups healed completely without scarring within 2 weeks with statistical significance. In both the treatment groups, new ulcers occurred throughout the follow-up period of 6 months. The mean number of the ulcers are statistically not significant with $p$ value $>0.05$.

Conclusion: Our study showed clinically beneficial effects with topical curcumin with regard to ulcer size, pain, healing, and recurrence rate. Also topical curcumin gel was well tolerated and performed ot par with topical triamcinolone acetonide oral paste with a borderline favorable result with triamcinolone.

Clinical relevance: Curcumin can be safely recommended on a long-term basis as a more appealing therapeutic agent and is a better alternative choice for aphthous ulcers in children, pregnant woman, lactating mother, and in immunocompromised individuals.

Keywords: Alternative treatment, Aphthous ulcers, Curcumin, Recurrent aphthous stomatitis, Topical steroid, Triamcinolone acetonide, Turmeric, Ulcer healing, Ulcer pain.

The Journal of Contemporary Dental Practice (2020): 10.5005/jp-journals-10024-2901
\end{abstract}

\section{INTRODUCTION}

Recurrent aphthous stomatitis (RAS, aphthae; canker sores) is the most common ulcerative condition of the oral mucosa characterized by multiple, recurrent, small, round, or ovoid ulcers with circumscribed margins, erythematous haloes, and yellow or gray floors that present first in childhood or adolescence and heals spontaneously with subsequent recurrence. ${ }^{1,2}$ The individual ulcers may occur in any form: minor, major, and herpetiform aphthae, with minor type being the most common one. ${ }^{1,2}$ In about $80 \%$ of participants with minor aphthous, the condition develops before the age of 30. A higher prevalence has been found in the higher socioeconomic groups, in females, and among individuals with stress, such as students at the time of examinations. ${ }^{3-5}$ Due to the indeterminate etiology of the disease, it is difficult to find a definitive cure, and current treatments are aimed toward ameliorating the symptoms. There are very limited published clinical evidence for the efficacy of the therapies used in this condition. The American Academy of Oral Medicine recommends topical treatment for aphthous ulcers. Topical corticosteroids have remained the mainstay of therapy for RAS. ${ }^{3}$

"Curcumin" (diferuloylmethane) is the principal curcuminoid of the popular Indian spice "Turmeric," which is a member of the ginger family. The curcuminoids are the polyphenols and are responsible for the yellow color of turmeric, first identified in 1910 by Lampe and Milobedzka. It is a common spice known mostly for its use in Indian dishes as a common ingredient in curries and other ethnic meals. Curcumin is as powerful as an antioxidant. Studies show that curcumin could downregulate the expression of interleukin
${ }^{1}$ Department of Oral Medicine and Radiology, Sathyabama Dental College and Hospital, Chennai, Tamil Nadu, India

2,3 Department of Oral Medicine and Radiology, SRM Dental College, Ramapuram, Chennai, Tamil Nadu, India

${ }^{4}$ Span Dental Care Centre, Mylapore, Chennai, Tamil Nadu, India

${ }^{5}$ Department of Oral Medicine and Radiology, Thai Moogambigai Dental College, Chennai, Tamil Nadu, India

Corresponding Author: Praveena Raman, Department of Oral Medicine and Radiology, Sathyabama Dental College and Hospital, Chennai, Tamil Nadu, India, Phone: +91 7373230303, e-mail: drpraveena7@gmail.com

How to cite this article: Raman P, Pitty R, Krithika CL, et al. Topical Curcumin and Triamcinolone Acetonide in Recurrent Minor Aphthous Ulcers: A Pilot Trial. J Contemp Dent Pract 2020;21(8): 884-890.

Source of support: Nil

Conflict of interest: None

6 (IL-6), tumor necrosis factor (TNF), and various other chemokines that could suppress inflammation through multiple pathways. ${ }^{6,7}$ It is used in various systemic conditions and has also been tried in oral mucosal conditions such as oral submucous fibrosis (OSMF), oral lichen planus (LP), and oral precancers. ${ }^{8}$ Besides its varied beneficial and safer effects in humans, curcumin has not been extensively investigated in the management of aphthous ulcers. Instead of using a topical anesthetic or an anti-inflammatory agent, 
a completely safer and a naturally occurring agent can be used as an alternative for the symptomatic relief of aphthous ulcers.

Corticosteroids have been in regular clinical usage for a range of inflammatory and immune-mediated conditions for over 50 years. Triamcinolone acetonide is one of the most commonly used medium potency topical steroid in RAS with relatively good results apart from its other varied indications such as OSMF, LP, Burning mouth syndrome, eczema, psoriasis, allergies, and so on. Triamcinolone acetonide $0.1 \%$ is used as a topical application, 2 to 3 times a day, for the palliative treatment of aphthous ulcers. The side effects of corticosteroids are dose related, and topical steroids are generally well tolerated. ${ }^{9,10}$

Previously conducted studies show limited evidence to significantly substantiate the clinical efficacy of topical curcumin and topical triamcinolone acetonide in the management of aphthous ulcers based on various clinical variables. To our knowledge, this single-center, interventional, blinded, parallel comparison trial is the first of its kind conducted to evaluate the efficacy of topical curcumin gel and topical triamcinolone acetonide oral paste in recurrent minor aphthous ulcers in a professional student population with a followup period of 6 months by assessing 6 clinical variables that include site of the ulcer, size of the ulcer, ulcer pain, healing period, frequency of recurrence, and number of ulcers, studied with appropriate statistical analysis. In this context, this study is an important addition to the existing literature.

\section{Materials and Methods}

This study was designed and carried out at Department of Oral Medicine and Radiology, SRM Dental College and Hospital, Ramapuram, Chennai, Tamil Nadu, and was approved by the Institutional Ethical and Scientific Committee Board. The procedures followed were in accordance with the ethical standards of the responsible committee on human experimentation (institutional or regional) and with the Helsinki Declaration of 1975 that was revised in 2000 .

The sample constitutes the students from the study institution. Sample size was calculated with $95 \%$ confidence interval and an error rate of $\pm 5 \%$, determining the need to include 30 participants in this pilot trial. Initially, a pro forma was prepared with five short questions (episodes and previous experience to oral ulcers, recurrence rate of their oral ulcers, number of ulcer episodes they get and regarding treatment history of ulcers) and was distributed to all undergraduates and postgraduates, following which 670 pro formas were received and evaluated.

Single or multiple minor aphthous ulcers, round or oval shaped, shallow with regular margins, surrounded by an erythematous halo and a yellowish base, variable in size but less than $10 \mathrm{~mm} / 1 \mathrm{~cm}$ in diameter, and occurring at nonkeratinized oral mucosa were only included in the study. Participants should have a minimum of at least two episodes of ulcers within the past 6 months to enter the trial, as the study period requires a minimum of 6 months (parallel comparison study). Subjects with age-group between 18 years and 30 years were only included in the study. Self-limitation of the oral ulcers (history of spontaneous healing without scarring, sequel, and medications) and participants with each episode of ulcers lasting from few days to a maximum of 2 weeks were only included on the study. Clinical confirmation of minor aphthous ulcers was done by two oral medicine experts following the World Health Organization guidelines.
Based on Figure 1, participants under any other form of treatment for their oral ulcers, those under orthodontic treatment; those with a known history of any systemic disorder such as anemia (excluded by routine hematological tests under consent); or if their oral ulcers are associated with ocular, dermal, genital, or gastrointestinal symptoms; those on medications such as NSAIDs, chemotherapeutic drugs, or with a history of systemic immunosuppressives; those with a known history of hypersensitivity to foods or drugs, history of tobacco, and alcohol usage in the recent 6 months period; pregnancy; and lactating woman were all excluded from the study. From the total of 670 pro formas, 601 pro formas met one or more of the exclusion criteria. The remaining 69 subjects were randomized by computer software and allocated to 2 groups: 34 participants in group I and 35 participants in group II. Group I participants were treated with topical curcumin-"Curnext": Curcuma longa $10 \mathrm{mg}$ oral gel $2 \%$ and group II participants were treated with topical triamcinolone acetonide-“"Kenacort": triamcinolone acetonide oral paste $0.1 \%$. All the participants were informed to report to the study investigator on day 1 of their next episode of oral ulcer for clinical evaluation.

The investigator informed the willing participants in detail regarding the nature of the study and its possible consequences. To minimize the patient dropouts, only those students who were willing to participate, willing to get a cure for their ulcer episodes, and willing to cooperate throughout the study period were selected, and no one was forced to accept. Privacy of all the study participants was ensured. Each participant had the right to withdraw at any time during the study.

Based on all the inclusion criteria, on day 1, site of the ulcer, size of the ulcer, baseline pain score, and number of ulcers were recorded by the investigator. The size of the ulcer was measured using a sterile calibrated William's periodontal probe and the longest diameter was recorded. As a rule, only symptomatic ulcers were treated. The start of the treatment was counted as day 1 which is also the first day of appearance of the ulcer. Group I and group II Participants were provided with the same amount of topical curcumin and triamcinolone, respectively, in an identically packed containers on day 1 of the appointment. The study investigator delivered the drug to each participant. In both the groups, the participants were blinded to the drug which they received. Ulcer pain was evaluated based on a combination of numeric pain rating scale and verbal pain intensity scale from 0 to $5 \mathrm{~cm} ; 0-$ No pain, 1-Mild pain, 2-Moderate pain, 3-Severe pain, 4-Very severe pain, and $5-$ Worst pain, according to the National Initiative on Pain Control ${ }^{\mathrm{TM}}$ (NIPC ${ }^{\mathrm{TM}}$ ).

Investigator supervised the first application of the drug and provided the subject with verbal and written instructions on how to apply the drug as shown in Figure 2A. Observations were carried out in a Log diary that was provided as sheets of papers to each participant at the end of day 1 of appointment, as per Figure 2B. Participants were instructed to complete one diary sheet per day until their ulcer heals. They were thoroughly followed on WhatsApp remainder every weekend and a day before their next clinical appointment. Participants were recalled every third day, fifth day, seventh day, and the day after healing to evaluate for symptomatic reduction in pain, size, occurrence of any new ulcers, and reduction in the number of ulcers and ulcer healing. Figures $3 \mathrm{~A}$ and $\mathrm{B}$ show clinical picture before and after treatment with curcumin, respectively. Figures $3 C$ and $D$ show clinical picture before and after treatment with triamcinolone, respectively. Completed log 


\section{DEPARTMENT OF ORAL MEDICINE AND RADIOLOGY-QUESTIONNAIRE FORM}

Name/sex /age/year of the study:

1) Have you ever suffered from oral ulcers?
a) Yes
b) No
c) Don't know

2) If yes, at what age your first ulcer occurred?
a) $<5 \mathrm{yrs}$
d) $15-20 \mathrm{yrs}$
b) $5-10$ yrs
e) $>20$ yrs
c) $10-15 \mathrm{yrs}$

3) How frequently do you get ulcer episodes?
a) Every month
c) Every 6 months
b) Every 3 months
d) Once a year

4) How many numbers of ulcers occurred at a given time?
a) Single
b) Multiple

5) Are your ulcers symptomatic? a)Yes b)No

6) If yes, have you ever taken any treatment for your ulcers? a)Yes b)No

7) If yes, what treatment do you often choose?
a) Self-medication
c) As per doctors advice
b) Herbal
d) Other

8) Where do the oral ulcers occur?
a) Labial mucosa: Upper/ Lower
d) Tongue
b) Buccal mucosa
e) Palate
c) Floor of the mouth
f) Lip

9) For how many days does the ulcer persist?
a) Less than a week
c) Nearly a month
b) More than a week
d) More than a month

10) Any family history of oral ulcers? a)Yes b) No

11) Do you suffer from any medical problem? If yes, please mention.

12) If yes, are the above symptoms associated with oral ulcers?
a) Yes-Occasionally/ More often b)No

13) History of any medications? a)Yes b)No. If yes, please mention with duration.

14) History of smoking? a)Yes b)No

15) History of alcohol consumption? a)Yes b) No

16) Are your ulcers associated with stress? a)Yes b)No

17) If yes, what is the possible predictable reason for your stress?
a) Menstruation
c) Domestic issues
b) Examinations
d) Other reasons

18) Rate the average/usual severity of your ulcer pain.
a) No Pain
b) Mild
c) Moderate
d) Severe
e) Very severe f) Worst possible pain

19) Description of your ulcer in your own words:

Kindly report to the department on day 1 of your next ulcer for clinical assessment

Fig. 1: Study questionnaire: given to 138 participants who had frequent history of oral ulcers 
1. Apply the gel after drying the ulcer by gently patting it with a soft piece of clean cotton.

2. Squeeze out approximately $1 / 4$ inch $(0.5 \mathrm{~cm}$ - peanut size $)$ of the gel from the tube on a clean fingertip and apply gently once or twice to the site of the ulcer. Do not rub the area.

3. Apply the gel three times daily (after breakfast, after lunch and after dinner) until your pain completely subsides.

4. Do not consume any beverages for $15 \mathrm{~min}$ after the application of the drug.

5. Observations are to be carried out in the Log diary

6. Contact immediately on experiencing an allergic reaction or a mild irritation or a serious hypersensitivity reaction.

A

Name, Age/Sex:

Date:

Verbal pain intensity scale-from 0-5

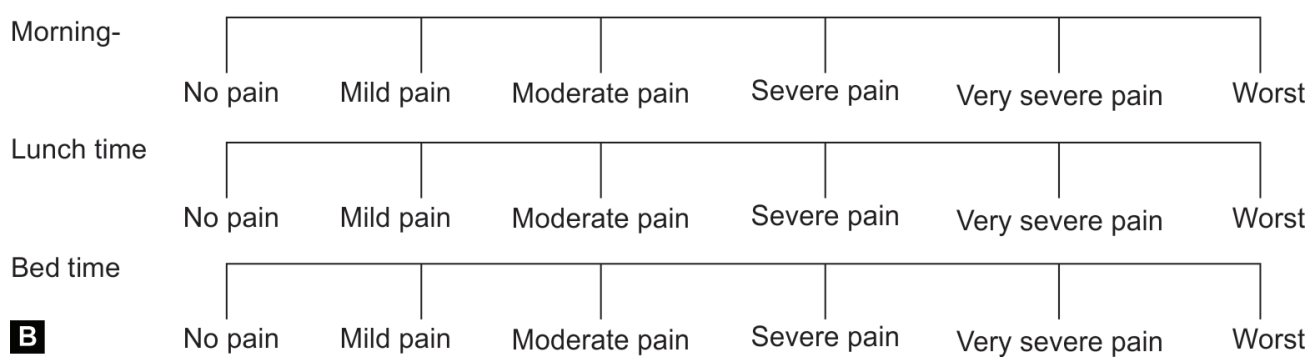

Figs 2A and B: Written details given to participants: (A) Shows the instructions given to the participants for applying their respective drug; (B) is the log diary given to note down their pain intensity during morning, lunch time and at bed time with verbal pain intensity scale


Figs 3A to D: (A) Before treatment with topical curcumin (group I); (B) After treatment with topical curcumin (group I); (C) Before treatment with topical triamcinolone acetonide (group II); (D) After treatment with topical triamcinolone acetonide (group II)

diaries were evaluated individually the next day once the ulcer heals. Participants' overall satisfaction was assessed at the end of the treatment. All the participants were followed up for a period of 6 months. Group I and group II participants were treated only with their respective drugs throughout the 6 month period. As a safety evaluation, a feedback was obtained from all the study participants. All the participants reported with good general health with no significant abnormalities before, during, or after the study.

Statistical analysis was performed using the SPSS (Statistical Package for the Social Sciences) software version 22.0 and significant level was accepted with $p$ value $<0.05$. Independent sample $t$ test was carried out to compare between the means and Chi-square test was performed to compare the proportions between the groups and Kaplan-Meier Survival Analysis. Log rank was performed to estimate the mean number of days required for ulcer pain reduction and ulcer healing.

\section{Results}

At the beginning of the trial, there were 34 participants in group I and 35 participants in group II. During the study period, three participants were lost to follow-up in group I, one participant from each group went to orthodontic treatment, and four participants discontinued the treatment in group II due to personal reasons. So, a total of nine dropouts was met. Therefore, the data from these nine participants were not considered for evaluation of the study outcome. Finally, 60 participants with recurrent minor aphthous ulcers completed the study within the study period.

The mean age (in years) was found to be 21.3 and 21.6 in group I and group II, respectively. The total number of males was found to be $11(36.7 \%)$ and 8 (26.7\%) and females was found to be 19 (63.3\%) and $22(73.3 \%)$ in group I and group II, respectively. Lower labial mucosa was found out to be the most common site of occurrence of aphthous ulcer in both the groups (33.8\% in group I and $32.5 \%$ in group II). Initially, the size of the ulcer was found to be increasing gradually in both the groups, followed by a significant reduction 
Table 1: Independent samples $t$ test to compare mean ulcer size $(\mathrm{cm})$ between group I and group II

\begin{tabular}{lllllr}
\hline Variables & Group & Mean & Std. Dev & t value & pvalue \\
\hline Ulcer size at day 1 & Group I & 0.213 & 0.0881 & 1.879 & 0.062 \\
Ulcer size at day 2 & Group II & 0.197 & 0.0512 & & \\
& Group I & 0.280 & 0.1140 & 2.133 & 0.034 \\
Ulcer size at day 3 & Group II & 0.257 & 0.0596 & & \\
& Group I & 0.363 & 0.1418 & 2.627 & 0.009 \\
Ulcer size at day 4 & Group II & 0.327 & 0.0814 & & \\
Ulcer size at day 5 & Group I & 0.401 & 0.1890 & 5.092 & $<0.001$ \\
& Group II & 0.308 & 0.1054 & & \\
Ulcer size at day 6 & Group I & 0.338 & 0.2103 & 3.808 & $<0.001$ \\
& Group II & 0.247 & 0.1477 & & 0.401 \\
Ulcer size at day 7 & Group I & 0.271 & 0.2345 & 0.844 & \\
& Group II & 0.180 & 0.2049 & & 0.499 \\
\hline
\end{tabular}

Table 2: Kaplan-Meier survival analysis for time (days) required for pain reduction: mean time (days) required for pain reduction and overall comparison

\begin{tabular}{|c|c|c|c|c|}
\hline \multirow[b]{3}{*}{ Group } & \multicolumn{4}{|c|}{ Mean } \\
\hline & \multirow[b]{2}{*}{ Estimate } & \multirow[b]{2}{*}{ Std. error } & \multicolumn{2}{|c|}{$95 \%$ confidence interval } \\
\hline & & & $\begin{array}{l}\text { Lower } \\
\text { bound }\end{array}$ & $\begin{array}{l}\text { Upper } \\
\text { bound }\end{array}$ \\
\hline Group I & 5.310 & 0.065 & 5.182 & 5.438 \\
\hline Group II & 4.570 & 0.063 & 4.446 & 4.695 \\
\hline \multirow[t]{2}{*}{ Overall } & 4.938 & 0.052 & 4.837 & 5.040 \\
\hline & Chi-square & $d f$ & \multicolumn{2}{|r|}{$p$ value } \\
\hline $\begin{array}{l}\text { Log rank } \\
\text { (Mantel-Cox) }\end{array}$ & 53.834 & 1 & \multicolumn{2}{|r|}{$<0.001$} \\
\hline
\end{tabular}

with a lower mean value in group II while compared to group I. Statistical significant difference was observed at second day, third day, fourth day, and fifth day in both the groups with a lower mean value in group II while compared to group I, inferring that both the drugs reduced the size of the ulcer but Drug $B$ is able to reduce the ulcer size little earlier than Drug A (Table 1).

A gradual reduction in pain was assessed in both the groups. Statistically significant difference was observed at first day, second day, third day, fourth day, and fifth day with $p$ value $<0.05$. A reduction in mean value in group II infers that both Drug $A$ and Drug $B$ are able to bring down the pain scores but Drug $B$ is able to reduce pain little faster than Drug A. Kaplan-Meier survival analysis (Table 2 and Fig. 4) estimated the mean time (days) required for pain reduction in group I as 5.310 (95\% confidence interval 5.182, 5.438) and in group II as 4.570 (95\% confidence interval 4.446, 4.695), and it is statistically significant in Log rank (Mantel-Cox) with $p$ value $<0.001$ meaning that Drug $A$ takes an average of 5.310 days for pain reduction and Drug $B$ takes an average of 4.570 days for pain reduction (slightly earlier than Drug A).

All the ulcers in both the groups healed completely without scarring within 2 weeks. Kaplan-Meier survival analysis estimated the mean time (days) required for ulcer healing in group I as 7.212 (95\% confidence interval 7.054, 7.370) and in group II as $6.769(95 \%$ confidence interval $6.604,6.935)$, and it is statistically significant
Table 3: Kaplan-Meier survival analysis for mean time (days) required for ulcer healing and overall comparison

\begin{tabular}{|c|c|c|c|c|}
\hline \multirow[b]{3}{*}{ Group } & \multicolumn{4}{|c|}{ Mean } \\
\hline & \multirow[b]{2}{*}{ Estimate } & \multirow[b]{2}{*}{ Std. Error } & \multicolumn{2}{|c|}{$95 \%$ confidence interval } \\
\hline & & & $\begin{array}{l}\text { Lower } \\
\text { bound }\end{array}$ & $\begin{array}{l}\text { Upper } \\
\text { bound }\end{array}$ \\
\hline Group I & 7.212 & 0.081 & 7.054 & 7.370 \\
\hline Group II & 6.769 & 0.084 & 6.604 & 6.935 \\
\hline \multirow[t]{2}{*}{ Overall } & 6.991 & 0.060 & 6.874 & 7.109 \\
\hline & Chi-square & $d f$ & \multicolumn{2}{|r|}{$p$ value } \\
\hline $\begin{array}{l}\text { Log rank } \\
\text { (Mantel-Cox) }\end{array}$ & 13.385 & 1 & \multicolumn{2}{|r|}{$<0.001$} \\
\hline
\end{tabular}

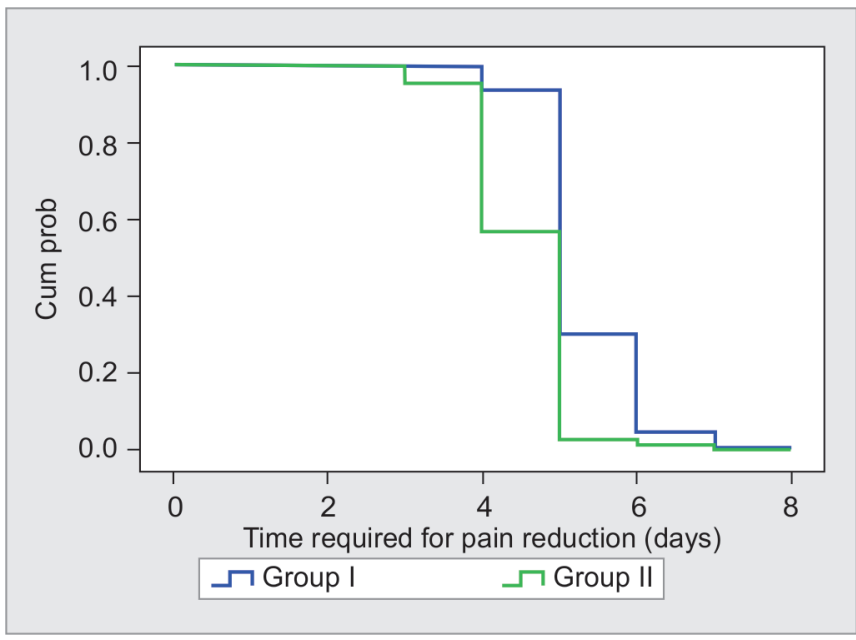

Fig. 4: Survival curve for time (days) required for pain reduction

in Log rank (Mantel-Cox) with $p$ value $<0.001$, inferring that Drug A takes an average of 7.212 days for healing and Drug $B$ takes an average of 6.769 days for ulcer healing (slightly earlier than Drug A) (Table 3 and Fig. 5). In both the treatment groups, new ulcers occurred throughout the follow-up period of 6 months. 


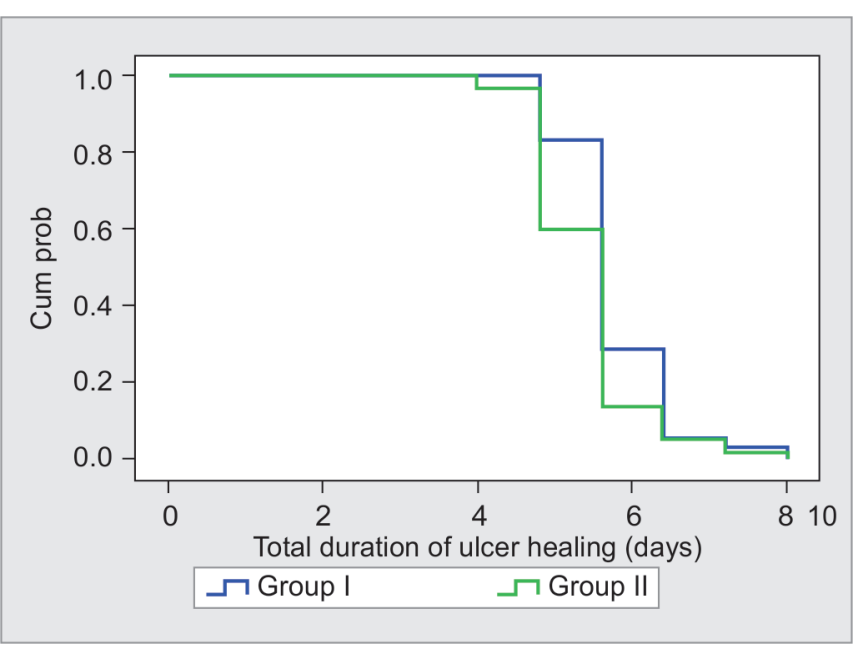

Fig. 5: Survival curve for healing time

The mean recurrence days is 54.34 and 60.66 in group I and group II, respectively, with a higher mean value in group II indicating more recurrence of ulcers in group II. The two means are not statistically significant ( $p$ value $>0.05$ ), meaning that irrespective of the drug which is being used, the ulcers do recur with the use of both the drugs and the rate of recurrence is approximately one week late in group II than in group I. Statistical significance was not observed with respect to number of ulcers.

There were no major adverse effects reported in both the groups during the study period. However, very few participants reported a feeling of chillness on application of curcumin, but still they continued applying the drug. All the participants irrespective of the age or gender were satisfied with their respective drug.

\section{Discussion}

Greer et al. proposed that the treatment effects in oral aphthous ulcer is caused by inhibition of histamines and leukotrines in the oral mucosa, although the exact working mechanism is still unclear. ${ }^{11}$ Scully stated that the cyclic nature of RAU makes it difficult to conduct a well-designed prospective double-blind controlled clinical trial, as some participants may have mild outbreaks, while others may have longer episodes or in combination with difference in size, number, and frequency of ulcer occurrence. Thus, the nature and severity of the recurrent aphthous ulcers varies and all these factors contribute to the inconsistency for a definite management of aphthous ulcers. ${ }^{12}$ Over the last decade, a wide range of medicinal plant parts are extracted and used as raw drugs, and they possess varied medicinal properties. As both the drugs used in this study are in the same topical route of administration, it is pharmacologically ideal to compare the efficacy. A parallel design was chosen with a follow-up period of 6 months due to a limited study period among a student population and to minimize patient drop out. Natha has proposed a set of criteria's to diagnose recurrent aphthous ulcerations. ${ }^{13}$ As the present study is set up in an educational institution where students were enrolled to participate, only few criteria from the Nathas criteria were taken into consideration to diagnose aphthous ulcers, and moreover the confirmation was done by two Oral Medicine experts.

The mean age in the present study was 21.45 years which is in accordance with the previous studies conducted by Byahatti ${ }^{3}$ and Arun Kumar et al. ${ }^{4}$ and not in accordance with the studies conducted by Gallo et al. and Deshmukh et al., ${ }^{14}$ as in these studies, selection criteria for age was not fixed prior to the study. Pongissawaranun et al. conducted a study on Thai population and found the incidence of RAS to be the maximum around $20-30$ years of age. ${ }^{15}$ In $80 \%$ cases of minor aphthous ulcers, the occurrence is predominantly observed in $<30$ years. ${ }^{1-5}$ The reason for the increased prevalence in professional students is thought to be stress, which is associated with the pressure of academics, demonstrating that stress plays a major role in precipitation. Literature review suggest the role of stress as an environmental trigger for the occurrence of aphthous ulcers. ${ }^{16}$ Hence the present study had recruited participants from an ideal professional dental college student population from 18 to 30 years in who the prevalence of aphthous ulcers and the period of stress is more.

In the present study, female predominance was observed in both the groups which is in accordance with the studies conducted by Nolan et al., ${ }^{17}$ Malayil et al., ${ }^{18}$ Abdullah, ${ }^{19}$ Byahatti, ${ }^{3}$ Arun Kumar et al., ${ }^{4}$ Pongissawaranun et al. ${ }^{15}$ and Leonardo et al. ${ }^{20}$ and not in accordance with McCullough et al. ${ }^{21}$ and Bhat et al. ${ }^{22}$ McCollough et al. found a significant relation to menstruation and aphthous ulcers. The possible explanation that can account for the higher female predominance in our study may be due to higher number of females in each academic batch.

In our study, lower labial mucosa was found to be the most prevalent site which is in accordance with the earlier studies conducted by Malayil et al. ${ }^{18}$, Bhat et al. ${ }^{22}$, and Abdullah ${ }^{19}$ but not in accordance with the study conducted by Arun Kumar et al. ${ }^{4}$ In our study, triamcinolone was able to reduce the ulcer size earlier than Curcumin which is in accordance with the previously conducted randomized clinical trial by Deshmukh et al. ${ }^{14}$ and not in accordance with the randomized trial conducted by Halim et al. ${ }^{23}$

In our study, pain of the ulcer was evaluated by the subjects based on a combination of numeric pain rating scale and verbal pain intensity scale from 0 to $5 \mathrm{~cm}$ according to the National Initiative on Pain Control ${ }^{\mathrm{TM}}$ (NIPC ${ }^{\mathrm{TM}}$ ) for easy perseverance which is also less time consuming, which no other previous studies have followed. In the present study, a lower mean value for pain was observed in triamcinolone group, inferring that both curcumin and triamcinolone were able to bring down the pain scores, but triamcinolone is able to reduce pain a little faster than curcumin which is in accordance with Halim et al. ${ }^{23}$ and Deshmukh et al. ${ }^{14}$

Our study was the first of its kind to perform a survival analysis, which allows for a graphical comparison of survival curves for both the treatment groups. Based on the survival analysis, curcumin and triamcinolone takes an average of 5.310 days and 4.570 days, respectively, for pain reduction and 7.212 days and 6.769 days, respectively, for ulcer healing with $p$ value $<0.001$. Hence, these data cannot be compared to any previous trials. In our study, the rate of healing of ulcers in group II was little faster than in group I which is not in accordance with Deshmukh et al. ${ }^{14}$ Few possible explanations that can counter the difference in healing time may be the individual's response to external stimuli. Pain expression may differ among individuals. Also, participants could have missed the application of their drug during morning, lunch time, or at bed time due to work pressure or domestic issues which is difficult to monitor. In our study, both the treatment groups had episodes of new ulcers occurring throughout the follow-up period of 6 months with a mean recurrence days of 54.34 and 60.66 in curcumin group and triamcinolone group, respectively, with $p$ value $>0.05$ meaning that irrespective of the drug that is being used, the aphthous ulcers 
do recur, but the recurrence is approximately 6 days delayed with triamcinolone. These data from our study cannot be compared to the previous trials using curcumin and triamcinolone as our study is the first trial to measure recurrence as an outcome. In the present study, there was no decline observed in the number of ulcers throughout the 6 month follow-up period using both the drugs, with $p$ value $>0.05$ which is in accordance to the studies conducted by Deshmukh et al. ${ }^{14}$ and Nolan et al. ${ }^{17}$

\section{LiMITATIONS}

Sample size and the study period were compromised, as the study was institutional based. Nature of the sample was not uniform or hemogeneous, as few participants especially postgraduates were from different parts of India. Probably a large cohort, randomized controlled studies for a longer follow-up period with no dropouts, possibly using a double-blind or a triple-blinded, crossover design along with the evaluation of immunological markers, could maximize the sensitivity of the therapeutic efficacy of curcumin in aphthous ulcer.

\section{Conclusion}

Till date, there is no cure/one drug treatment for aphthous ulcers. Long-term use of topical steroids possess risk of changes in oral flora leading to mucosal atrophy, hypersensitivity reactions, and oral candidiasis. Topical curcumin, being a herbal analgesic and anti-inflammatory, can be safely recommended and is a better alternative choice in children, pregnant woman, lactating mother, and immunocompromised individuals.

\section{ACKNOWLeDgments}

The author would like to remember and thank all the study participants for their cooperation in the study; Dr P Venkatesan MSc, MPhil, MPS, PDCDM, PhD and Mr Boopathi MSc, MBA for their valuable statistical assessments, analysis and counseling in arriving at the study results; and Mr Karthikeya Murthy, Mrs and Mr Dr K Raman and Mrs and Mr Jagannathan, for their constant support and ever-willingness to guide for the manuscript writing and data compilation.

\section{References}

1. Tappuni AR, Kovacevic T, Shirlaw PJ, et al. Clinical assessment of disease severity in recurrent aphthous stomatitis. J Oral Pathol Med 2013;42(8):635-641. DOI: 10.1111/jop.12059.

2. Liang MW, Neoh CY. Oral aphthosis: management gaps and recent advances. Ann Acad Med Singapore 2012;41(10):443-470.

3. Byahatti SM. Incidence of recurrent aphthous ulcers in a group of student population in libya: a questionnaire study. Arch CranOroFac Sci 2013;1(2):26-30.

4. Arun Kumar M, Ananthakrishnan V, Goturu J. Correlation of ulcer size and serum cortisol in subjects with aphthous ulcers. Int J Sci Res 2013;2(11):381-382.

5. Sharma M, Gupta R, Singh S. Correlation of psychological stress with recurrent aphthous stomatitis among dental students in an educational institution. Int J Appl Dent Sci 2017;3(4): 455-458.

6. Nita C-W. Safety and anti inflammatory activity of curcumin: a component of turmeric (Curcuma longa) J Altern Complement Med 2003;9(1):161-168. DOI: 10.1089/107555303321223035.

7. Shrishai $D$, Harish $H$, Ravichandra $H$, et al. Turmeric: nature's precious medicine. Asian J Pharm Cl in Res 2013;6(3):10-16.

8. Lawande SA. Therapeutic applications of turmeric in dentistry: a promising future. J Pharm Biomed Sci 2013;27(27):586-591.

9. Belenguer-Guallar I, Jiménez-Soriano Y, Claramunt-Lozano A. Treatment of recurrent aphthous stomatitis. A literature review. J Clin Exp Dent 2014;6(2):e168-e174. DOI: 10.4317/jced.51401.

10. González-Moles MA. The use of topical corticoids in oral pathology. Med Oral Pathol Oral Cir Buccal 2010;1(15):e827-e831. DOI: 10.4317/ medoral.15.e827.

11. Greer Jr RO, Lindenmuth JE, Juarez T, et al. A double-blind study of topically applied 5\% amlexanox in the treatment of aphthous ulcers. J Oral Maxillofac Surg 1993;51(3):243-248. DOI: 10.1016/S02782391(10)80164-8.

12. Scully C. Clinical practice. aphthous ulceration. N Engl J Med 2006;355(2):165-172. DOI: 10.1056/NEJMcp054630.

13. Natah SS, Konttinen YT, Enattah NS, et al. Recurrent aphthous ulcers today: a review of the growing knowledge. Int J Oral Maxillofac Surg 2004;33(3):221-234. DOI: 10.1006/ijom.2002.0446.

14. Deshmukh RA, Bagewadi AS. Comparison of effectiveness of curcumin with triamcinolone acetonide in the gel form in treatment of minor recurrent aphthous stomatitis: a randomized clinical trial. Int J Pharm Investig 2014;4(3):138-141. DOI: 10.4103/2230-973X.138346.

15. Pongissawaranun W, Laohapand P. Epidemiologic study on recurrent aphthous stomatitis in a thai dental patient population. Community Dent Oral Epidemiol 1991;19(1):52-53. DOI: 10.1111/j.1600-0528.1991. tb00106.x.

16. Huling LB, Baccaglini L, Choquette L, et al. Effect of stressful life events on the onset and duration of recurrent aphthous stomatitis. J Oral Pathol Med 2012;41(2):149-152. DOI: 10.1111/j.1600-0714.2011.01102.x.

17. Nolan A, Baillie C, Badminton J, et al. The efficacy of topical hyaluronic acid in the management of recurrent aphthous ulceration. J Oral Pathol Med 2006;35(8):461-465. DOI: 10.1111/j.16000714.2006.00433.x.

18. Malayil S, Thomas J, Rani Mol P, et al. Frequency of patients presenting with recurrent aphthous stomatitis: A pilot study. J Dent Med Sci 2014;13(1):63-66. DOI: 10.9790/0853-13186366.

19. Abdullah J. Prevalence of recurrent aphthous ulceration experience in participants attending piramird dental speciality in Sulaimani city. J Clin Exp Dent 2013;5(2):e89-e94. DOI: 10.4317/jced.51042.

20. Leonardo ML, Diogo MD, Arnaldo Jr FC, et al. Prevalence and risk factors for the development of RAS. Rev Cir Traumatol Buco-Maxilofac Camaragibe 2010;10(2):61-66.

21. McCullough MJ, Abdel-Hafeth S, Scully C. Recurrent aphthous stomatitis revisited; clinical features, associations, and new association with infant feeding practices. J Oral Pathol Med 2007;36(10):615-620. DOI: 10.1111/j.1600-0714.2007.00589.x.

22. Bhat S, Sujatha D. A clinical evaluation of $5 \%$ amlexanox oral paste in the treatment of minor recurrent aphthous ulcers and comparison with the placebo paste: a randomized, vehicle controlled, parallel, single centre clinical trial. Indian J Dent Res 2013;24(5):593-598. DOI: 10.4103/0970-9290.123382.

23. Halim D, Abdul Khalik NIB, Taib $\mathrm{H}$, et al. Novel material in the treatment of minor oral recurrent aphthous stomatitis. Int Med J 2013;20(3):392-394. 